\title{
PAUBAHAN TEGES KOSA KATA BASA BALI KUNA SAJERONING BASA BALI BARU
}

\author{
I Ngh. Yoga Darma. AP ${ }^{1}$, I Kt. Paramarta ${ }^{1}$, I. Wyn. Gd. Wisnu ${ }^{2}$ \\ Jurusan Pendidikan Bahasa Bali \\ Universitas Pendidikan Ganesha \\ Singaraja, Indonesia \\ e-mail: \{darma.yoga@yahoo.com, ketut.paramarta@undiksha.ac.id, \\ wisnukawiswara@gmail.com\}@undiksha.ac.id
}

\begin{abstract}
KUUB
Tetilikan puniki matetujon mahbahang (1) Teges kruna basa Bali Kuna sajeroning prasasti warsa 915-942 Masehi miwah warsa 951-983 Masehi, (2) Paubahan tegse kruna basa Bali Kuna sajeroning basa Bali Baru. Jejering ring tetilikan puniki inggih punika prasasti mabasa Bali Kuna saking warsa 915-942 Masehi miwah warsa 951-983 Masehi. Panandang tetilikan puniki inggih punika paubahan teges kosa kata basa Bali Kuna sajeroning basa Bali Baru. Teori sane kanggen sajeroning tetilikan puniki inggih punika (1) teori semantic miwah (2) teori sosiolinguistik. Kramaning tetilik sane kaanggen inggih punika kramaning dokumentasi. Data tureksa sane kaanggen sakadi indentifikasi data, reduksi data, klasifikasi data, interpretasi data miwah panyutetan. Pikolih tetilikan puniki minakadi, (1) teges kruna basa Bali Kuna sajeroning prasasti warsa 915-942 Masehi miwah warsa 951-983 Masehi, saha nyantenang tegesnyane soang-soang, minakadi 95 kruna sane mateges tetep, 24 kruna sane tegesnyane magentos utawi berubh total, miwah 53 kruna sane mateges meluas miwah menyempit. (2) Paubahan teges kosa kata basa Bali Kuna sajeroning basa Bali Baru marupa 47 kruna madue paubahan teges meluas, miwah 6 kruna sane madue paubahan teges menyempit.
\end{abstract}

Kruna Jejaton: paubahan, teges, basa Bali Kuno, basa Bali Baru

\begin{abstract}
ABSTRAK
Penelitian ini bertujuan untuk menjelaskan: (1) Arti kata Bahasa Bali Kuna yang ada pada prasasti tahun 915-942 Masehi dan tahun 951-983 Masehi, (2) Perubahan makna Bahasa Bali Kuna dalam Bahasa Bali Baru. Subjek penelitian ini adalah prasasti berbahasa Bali Kuna dari tahun 915-942 Masehi dan tahun 951-983 Masehi. Objek penelitian ini adalah perubahan makna Bahasa Bali Kuna dalam Bahasa Bali Baru.Teori yang di gunakan pada penelitian ini adalah (1) teori semantic, (2) teori sosiolinguistik, dan (3) teori terjemahan. Metode yang digunakan pada penelitian ini yaitu metode dokumentasi. Analisis data yang digunakan dalam penelitian ini adalah indentifikasi data, reduksi data, klasifikasi data, interpretasi data dan kesimpulan. Hasil pada penelitian ini adalah (1) Arti kata Bahasa Bali Kuna pada prasasti tahun 915-942 Masehi dan tahun 951-983 Masehi, dan artinya masing-masing yang berupa 95 kata yang memiliki arti tetap, 24 kata yang meiliki arti berubah total, dan 53 kata yang memiliki arti meluas dan menyempit, (2) Perubahan makna kata Bahasa Bali Kuna dalam Bahasa Bali Baru, yang berupa 47 kata yang memiliki perubahan makna meluas dan 6 kata yang memiliki perubahan makna menyempit.
\end{abstract}

Kata Kunci: perubahan, makna, basa Bali Kuna, basa Bali Baru 


\begin{abstract}
This study aims to explain: (1) Meaning of the ancient Balinese language that is on the inscription of 915-942 AD and 951-983 AD, (2) The change of meaning of ancient Balinese language in new Balinese language. The subject of this study is an ancient Balinese language inscription from 915-942 AD and 951-983 AD. The object of this study is the change of meaning of ancient Balinese Language in New Balinese Language. The theories used in this study are (1) Semantic theory, (2) Sociolinguistic theory, and (3) Translation theory. The method that used in this research is documentation method. Data analysis used in this research is data identification, data reduction, data classification, data interpretation and conclusion. The results of this study are (1) The meaning of the ancient Balinese language on the inscription of 915-942 AD and 951-983 AD, then the meaning of each word is 95 words which have a fixed meaning, 24 words that have meaning changed completely, and 53 words that have a broad and narrow meaning, (2) The change of the meaning of the ancient Balinese language in the new Balinese language, which is 47 words that have a change of meaning extends and 6 words that have a narrowed meaning.
\end{abstract}

Keywords: changing, meaning, the ancient balinese language, the new balinese language

\section{PURWAKA}

Basa Bali pinaka silih sinunggil basa ring panegara Indonesia sane kanggen mabebaosan antuk krama Bali. Basa Bali nyihnayang kasujatian Bali sane madue budaya kaloktah ka dura negara. Kawigunan basa Bali taler nenten prasida lempas saking kawentenan yadnya sane kalaksanayang ring Bali, minakadi antuk para pinandita utawi pandita ri kala nguncarang puja mantra miwah para prawartaka karya ring yadnya punika. Basa Bali taler kanggen ri kala masimakrama, paruman adat, sekaa truna-truni, suka duka, paruman subak, miwah sane lianan. Punika nyihnayang basa Bali puniki banget mawiguna tur pinaka basa kapertama miwah utama sane kanggen ring Bali.

Basa Bali pinaka basa kapertama sampun wenten wit saking nguni. Manut Bawa, dkk (1985: 21) basa Bali manut ring aabnyane kakepah dados tiga inggih punika basa Bali Baru, basa Bali Tengahan, basa Bali Kuna. Basa Bali Kuna sampun wenten wit saking mijilnyane prasasti Sukawana Al kantos ring penyeneng agung Raja Anak Wungsu (Bawa 2002: 16). Panglimbak jagat Bali nglantur ring aab Bali Tengahan ngawinan wenten panglimbak basa Bali Tengahan. Manut Bawa dkk, (1984: 48) basa Bali Tengahan nglimbak ri kala aab
Karatuan Gelgel kantos Karatuan Klungkung (1350-1710 M). Kalanturang panglimbak basa Bali wus Karatuan Klungkung kantos mangkin kawastanin basa Bali Baru.

Kawentenan basa Bali Baru aabe kadi mangkin tan prasida lempas ring kauripan para janane ring Bali. Basa Bali Baru ketah kanggen sajeroning mabebosan, pagubugan, miwah paruman adat. Basa Bali Baru taler pinaka bantang tradisi miwah adat Bali. Basa Bali Baru pinaka jalaran panglimbak kaweruhan janane saking basa Bali Kuna kantos basa Bali Baru. Panglimbak basa Bali Baru nenten prasida lempas saking panglimbak basa Bali Kuna. Sajeroning basa Bali Baru kantun nganggen makudang-kudang kosa kata basa Bali Kuna. Basa Bali Kuna punika ketah kapanggihin ring susastra, wacana lisan, miwah prasasti-prasasti Bali Kuna.

Madasar antuk pratiaksa riin, basa Bali Kuna sane kapanggihin ring prasasti Bali Kuna punika kasujatiannyane akeh taler kantun kanggen sajeroning mabebaosan ring basa Bali Baru. Indike punika prasida kacingak ring kruna tua sane mateges 'ento', sakewanten sajeroning basa Bali Baru kruna tua punika mateges 'lingsir'; wenten taler kruna tukad sane mateges 'sungai' taler ring basa Bali 
Baru tukad punika mateges 'sungai' sane wenten ring prasasti Srokodan.

Basa Bali Kuna sane kantun kanggen sajeroning basa Bali Baru wenten ring prasasti warsa 915-942 miwah 951-983 Masehi. Sajeroning prasasti inucap wenten 19 prasasti, minakadi (1) Srokodan, (2) Babahan I, (3) Sembiran Al, (4) Pengotan Al, (5) Batunja Al, (6) Dausa, Pura Bukit Indrakila Al, (7) Serai Al, (8) Dausa, Pura Bukit Indrakila BI, (9) Gobleg, Pura Batur A, (10) Sembiran B, (11) Manik Liu Al, (12) Manik Liu B, (13) Manik Liu C, (14) Manukaya, (15) Kintamani A, (16) Kintamani B (17) KIntamani C, (18) Sembiran All, miwah (19) Gobleg, Pura Desa II. Prasasti ring ajeng kawangun antuk kruna basa Bali Kuna sane kantun kaanggen kantos mangkin. Kawentenan kosa kata basa Bali Kuna sane kanggen ring basa Bali Baru madue paubahan teges.

Paubahan teges basa Bali Kuna ring basa Baru wenten meluas miwah menyempit. Kabaos paubahan teges meluas duaning teges ring basa Bali Kuna wenten asiki nanging teges ring basa Bali Baru langkungan teken asiki. Kabaos paubahan teges menyempit duaning teges ring basa Bali Kuna wenten kalih utawi tiga, nanging teges ring basa Bali Baru wenten asiki. Kruna sane ngranjing ring paubahan teges meluas sakadi 'aji' sane mateges 'bapa' ring basa Bali Kuna nanging ring basa Bali Baru mateges bapa, kaweruhan, aji (harga), miwah raja. Kruna sane ngranjing ring paubahan teges menyempit sakadi 'langkir' sane mateges 'wuku kaping 13' miwah pesengan jadma, nanging ring basa Bali Baru mateges wuku kaping 13 kemanten. Punika nyihnayang wenten paubahan teges kosa kata basa Bali Kuna sajeroning basa Bali Baru.

Kantos mangkin dereng wenten tetilikan ngeninin indik paubahan teges kosa kata basa Bali Kuna sajeroning basa Bali Baru punika. Akehan tetilikan ngeninin fonologi miwah morfologi basa Bali Kuna, sakadi tetilikan $\mathrm{Ni}$ Luh Sutjiati Beratha (Australian National University) sane mamurda 'Evolution Of Verbal Morphology In Balinese'. Tetilikan antuk Ida Wayan Oka Granoka dkk (Depdikbud Denpasar) sane marupa kamus bahasa Bali Kuna-Indonesia. Tetilikan I Wayan Bawa (2002) sane mamurda 'Sejarah Perkembangan Bahasa Bali', ngeninin indik kawentenan panglimbak basa Bali sane kabaos periodisasi basa Bali. Tetilikan saking $\mathrm{R}$. Goris (1967) sane mamurda 'Ancient History of Bali'. Tetilikan punika maosang indik lelintihan (sejarah) Bali Kuno, sane mahbahang miwah nyorohang indik prasasti Bali Kuna minakadi soroh prasasti yumu pakatahu miwah soroh prasasti duk aab dinasti Warmadewa.

Maduluran antuk punika pacang kalaksanayang tetilikan ring prasasti warsa 915-942 Masehi miwah warsa 951-983 Masehi ngeninin paubahan teges kosa kata basa Bali Kuna sajeroning basa Bali Baru.

Bantang pikobet tetilikan (1) napi teges kruna basa Bali Kuna sajeroning prasasti warsa 915-942 Masehi miwah warsa 951-983 Masehi? (2) sapunapi paubahan teges kosa kata basa Bali Kuna sajeroning basa Bali Baru?

Tetujon tetilikan puniki inggih punika (1) Mangda prasida nlatarang teges kruna Basa Bali Kuna ring prasasti warsa 915-942 Masehi lan warsa 951-983 Masehi (2) Mangda prasida nlatarang paubahan teges kruna Basa Bali Kuna ring Basa Bali Baru.

$\mathrm{Ri}$ sajeroning tetilikan puniki wenten kawigunannyane, minakadi kawigunan pamucuk lan kawigunan panglimbak sane kabahbahang sakadi ring sor.

Kawigunan pamucuk (teoritis) nyaratang mangda pikolih tetilikan puniki prasida ngaturang kaweruhan indik krunakruna Basa Bali Kuna sane kari kanggen ring Basa Bali Baru miwah panglimbaknyane prasida kanggen sajeroning paguron-guron basa Bali. Tiosan ring punika, tetilikan puniki prasida kanggen sepat siku-siku ri kala makarya tetilikan indik Basa Bali Kuna. Kawigunan Panglimbak (praktis) tetilikan puniki inggih punika (a) majeng ring panilik. Pikolih tetilikan puniki prasida pinaka widya anyar duaning ri tatkala perkuliahan nenten wenten paplajahan ngeninin indik Basa Bali Kuna tur prasida nguningin indik kawentenan Basa Bali Kuna sane kantun kanggen sajeroning Basa Bali Baru, (b) majeng ring pangwacen. Pinaka pangweruhan miwah wawasan ngeninin indik Basa Bali Kuna, (c) majeng ring panilik salanturnyane. Tetilikan ngeninin indik kosa 
kata basa Bali Kuna mangda prasida kalimbakang malih tur sayan katincapang, (d) majeng ring pangajah basa Bali. Pikolih saking tetilikan puniki prasida kadadosang sarana miwah sesuluh anggen ngwewehin kaweruhan para pangajah Basa Bali nguningin kosa kata basa Bali Kuna, duaning basa Bali Kuna nenten kaplajahin ring mata kuliah.

\section{KRAMANING TETILIK}

Kramaning tetilik prasida ngicen tata cara ri kala jagi nyawis sekancan pikobet sane wenten ring sajeroning tetilik, sumangdane prasida ngamolihang pikolih tetilikan. Ring kramaning tetilik punika prasida kawedarang indik (1) palihan tetilik. Palihan tetilik inggih punika strategi ring sajeroning ngatur tetilik mangda panilik prasida mikolihang data sane patut miwah sane manut ring karakteristik variable miwah tetujon tetilik. Indike puniki, tetilik puniki nganggen palihan deskriptif kualitatif santukan sampun manut antuk tatujon tetilik, sane nyihnayang indik paubahan teges kosa kata Basa Bali Kuna sajeroning Basa Bali Baru. (2) jejering miwah panandang tetilik. Jejering tetilikan sajeroning tetilikan puniki inggih punika Prasasti Bali Kuna saking warsa 915-942 Masehi lan warsa 951-983 Masehi. panandang tetilikan puniki inggih punika paubahan teges kosa kata Basa Bali Kuna Sajeroning Basa Bali Baru. (3) kramaning mupulang data. Kramaning mupulang data sane kanggen sajeroning tetilikan puniki marupa kramaning dokumentasi. (4) piranti tetilik. Piranti sane kanggen inggih punika piranti dokumentasi. (5) data tureksa. Pamargi data tureksa sane kalaksanayang sajeroning tetilikan puniki inggih punika a) indentifikasi data b) reduksi data c) klasifikasi data d) interpretasi data e) panyutetan.

\section{PIKOLIH MIWAH TETEPASAN}

Ring adyaya puniki jagi kabahbahang indik pikolih miwah tetepasan tetilikan. Indike puniki mapaiketan sajeroning bantang pikobet sane nyihnayang indik (1) teges kruna Basa Bali Kuna ring prasasti warsa 915-942 miwah warsa 951-983 Masehi saha (2) paubahan teges kruna Basa Bali Kuna inucap ring Basa Bali Baru.
Bebaosan inucap kajangkepin antuk implikasi sane midartayang indik paiketan miwah pikenoh pidaging tetilikan puniki majeng ring kawentenan mata kuliah sajeroning Prodi Pendidikan Bahasa Bali Undiksha.

(1) Ring pikolih tetilikan puniki kajantenang indik wilangan miwah teges kruna Basa Bali Kuna sajeroning prasasti nganutin warsa 915-942 Masehi miwah warsa 951-983 Masehi. Kawentenan prasati sajeroning warsa 915-942 Masehi marupa 9 prasasti, luiripun: (1) Serokodan, (2) Babahan I, (3) Sembiran AI, (4) Pengotan Al, (5) Batunja Al, (6) Dausa, Pura Bukit Indrakila AI, (7) Serai Al, (8) Dausa, Pura Bukit Indrakila BI (9) Gobleg, Pura Batur A. Kawentenan prasasti sajeroning warsa 951983 Masehi marupa 10 prasasti, luiripun: (1) Sembiran B, (2) Manik Liu AI, (3) Manik Liu BI, (4) Manik Liu C, (5) Manukaja ${ }^{8}$ ), (6) Kintamani A, (7) Kintamani B, (8) Kintamani C, (9) Sembiran All, (10) Gobleg, Pura Desa II.

Panureksan sajeroning prasasti 915942 Masehi prasida ngamolihang data marupa 243 kruna. Data puniki mawit saking 37 kruna ring prasati Serokodan, 22 kruna ring prasasti Babahan I, 32 kruna ring prasasti Sembiran Al, 17 kruna ring prasasti Pengotan Al, 33 kruna ring prasasti Batunja Al, 48 kruna ring prasasti Dausa, Pura Bukit Indrakila Al, 30 kruna ring prasasti Serai Al, 16 kruna ring prasasti Dausa, Pura Bukit Indrakila BI, 8 kruna ring prasasti Gobleg, Pura Batur A. Panureksa sajeroning prasasti 951-983 Masehi prasida ngamolihang data marupa 192 kruna. Data puniki mawit saking 31 kruna ring prasasti Sembiran B, 22 kruna ring prasasti Manik Liu Al, 20 kruna ring prasasti Manik Liu BI, 18 kruna ring prasasti Manik Liu C, 12 kruna ring prasasti Manukaja ${ }^{8}$ ), 17 kruna ring prasasti Kintamani $A, 8$ kruna ring prasasti Kintamani B, 13 kruna ring prasasti Kintamani C, 35 kruna ring prasasti Sembiran All, miwah 16 kruna ring prasasti Gobleg, Pura Desa II. Data prasasti inucap sane mawilang 455 kruna katureksain malih dados 172 kruna, duaning saking 19 prasasti punika wenten wangun kruna sane pateh wantah kasurat asiki.

Manut teori terjemahan, terjemahan inggih punika ngingsirang teges kruna 
saking wit basa ka basa tiosan (Hariyanto, 2003: 118). Sajeroning panureksan puniki kapolihang kruna-kruna basa Bali Kuna ring prasasti warsa 915-942 Masehi miwah warsa 951-983 Masehi akehnyane 172 kruna. Sajeroning 172 kruna punika katureksain antuk terjemahan leksikal, inggih punika teges kruna sane kapolihang ring kamus (Hariyanto, 2003). Indike punika sampun prasida kasinahang ring kamus basa Bali Kuna (Granoka dkk, 1984).

Sajeroning nyinahang teges krunakruna ring prasasti warsa 915-942 Masehi miwah warsa 951-983 Masehi, panilik taler nguratiang paiketannyane ring prasasti. Teges kruna-kruna punika patut kauratiang duaning wenten pabinayan konteks ring kamus miwah ring prasasti. Sakadi kruna 'aji' sane mateges 'bapa' miwah 'pesengan jadma' ring basa Bali Kuna aji punika ketah kaanggen ring pesengan utawi gelar Raja punika prasida kapanggihin ring punggelan prasasti Manik Liu Al "anugrahan sang ratu sri aji tabanendra warmmadewa" sane mateges anugrah saking Raja Sri Aji Tabanendra Warmmadewa.

Asapunika taler ring kruna 'Indra' sane mateges 'bhatara Indra' (Granoka, 1984) ring basa Bali Kuna ketah kaanggen ring wastan genah. Punika prasida kapanggihin ring punggelan prasasti Sembiran All "di julah, di indrapura" sane mateges ring desa Julah, ring Indrapura.

Kruna 'çukla' sane mateges 'sukla' (Granoka, 1984) ring basa Bali Kuna ketah kaanggen nyihnayang galah bulan kasurat prasasti inucap sakadi sane kapanggihin ring punggelan prasasti Sembiran Al "çukla pañcami" sane mateges tanggal lima ring bula paro terang sane sukla.

Kruna 'Sidi' sne mateges 'sidi' (Granoka, 1984) ring basa Bali Kuna ketah kaanggen nyihnayang genah sakadi sane kapanggihin ring punggelan prasasti Babahan I "hangganya danginan da hyang bangkyang sidi" sane mateges kantosnyane ida ring sisi kangin wates genah patapan di ptung.

Yening uratiang teges kruna-kruna ring prasasti mabasa Bali Kuna saking warsa 915-942 Masehi miwah warsa 951983 Masehi tur kasaihang tegesnyane sareng basa Bali Baru, kapanggihin soroh kruna-kruna: 1) Kruna-kruna sane tegesnyane tetep (95), 2) Kruna sane tegesnyane magentos tur nenten madue paiketan teges ring basa Bali Baru (24), 3) Kruna-kruna sane tegesnyane meluas (47), miwah 4) Kruna-kruna sane tegesnyane menyempit (6).

Ngeninin indik paubahan teges kosa kata basa Bali Kuna sajeroning basa Bali Baru sane wenten sajeroning prasasti mabasa Bali Kuna utaminnyane saking warsa 915-941 Masehi miwah 951-983 Masehi, kasorohang dados kalih inggih punika paubahan teges meluas lan paubahan teges sane menyempit. Saking tetilikan sane sampun kamargiang, kapolihang 53 data kruna sane marupa paubahan teges meluas miwah menyempit sajeroning prasasti mabasa Bali Kuno.

(2) Manut sakadi teori sosiolinguistik paubahan teges meluas inggih punika paubahan teges sane ngawinang kruna madue teges langkungan teken asiki (Chaer, 2004: 141). Sajeroning tetilikan puniki kapolihang kosa kata basa Bali Kuna sane tegesnyane meluas sajeroning basa Bali Baru minakadi, kruna sane wit tegesnyane asiki dados kalih wenten telung dasa dua, kruna sane wit tegesnyane asiki dados tiga wenten solas, kruna sane wit tegesnyane asiki dados patpat wenten kalih miwah kruna sane wit tegesnyane asiki dados lelima wenten asiki. Pikolih tetilikan sane sampun kamargiang, paubahan teges meluas akehan ring paubahan teges menyempit sajeroning prasasti mabasa Bali Kuna. Kruna basa Bali Kuna sane kari kaanggen sajeroning basa Bali Baru madue teges meluas wenten 47 kruna.

Duaning sane pacang katepasin wantah indik paubahan teges meluas miwah paubahan teges menyempit krunakruna sane tegesnyane tetep miwah magentos nenten katepasin.

Kruna 'Aji' sajeroning basa Bali Kuna mateges 'bapa' (Oka Granoka dkk, 1984). Sajeroning basa Bali Kuna kruna aji ketah kanggen antuk bhiseka utawi gelar Raja punika prasida kapanggihin ring punggelan prasasti Manik Liu Al "anugrahan sang ratu sri aji tabanendra warmmadewa". Sajeroning basa Bali Baru kruna 'Aji' mateges 'Aji (harga)', 'Bapa', 'Raja', 'Widya', miwah 'Lakar' (Anom dkk, 2014). Ring 
tetilikan Beratha (1992) nenten kapanggihin kruna aji, nanging wenten kruna ajin sane mateges harga, tur sampun ngranjing ring low level kaanutang ring BalineseIndonesian Dictionary 1990. Kruna Aji ring basa Bali Baru sane mateges harga ngranjing ring kruna mider, duaning rasa basanyane nenten alus miwah kasar.

Kruna 'Batek' sajeroning basa Bali Kuna mateges 'kedeng' (Granoka dkk, 1984). Sajeroning basa Bali Baru kruna 'Batek' mateges 'wantah' miwah 'kedeng' (Anom dkk, 2014). Kruna 'batek' sane mateges kedeng ketah kanggen ring Kabupaten Jembrana. Sajeroning tetilikan Beratha nenten kapanggihin kruna 'batek'.

Kruna 'Guru' sajeroning basa Bali Kuna mateges 'Guru' (Granoka dkk, 1984). Sajeroning basa Bali Baru kruna 'Guru' mateges 'Guru' miwah 'panggilan bapa' (Anom dkk, 2014). Panggilan bapa nganggen kruna guru puniki wantah kaanggen ring kulawarga Bhujangga.

Kruna 'Padma' sajeroning basa Bali Kuna mateges 'tunjung bang' (Granoka dkk, 1984). Sajeroning basa Bali Baru kruna 'Padma' mateges 'tunjung' (Anom dkk, 2014). Manut pratiaksa riin, tunjung ring basa Bali Baru akeh sorohnyane nanging ring basa Bali Kuna wantah kasinahang tunjung bang.

Kruna 'Sukla' sajeroning basa Bali Kuna mateges 'Sukla' (Granoka dkk, 1984). Sajeroning basa Bali Baru kruna 'Sukla' mateges 'Sukla' miwah 'Sperma' (Anom dkk, 2014). Sajeroning tetilikan Beratha nenten kapanggihin kruna 'Sukla' duaning nenten wenten paubahan morfologinyane.

Kruna basa Bali Kuna sajeroning basa Bali Baru lianan ring madue paubahan teges sane meluas taler madue paubahan sajeroning sor singgih basa, duaning ring basa Bali Kuna dereng prasida kajantenang kawentenan sor singgih basa (Beratha, 1992). Ring basa Bali Kuna kapanggihin kruna 'duka' sane mateges 'sungsut', kruna punika ring basa Bali Kuna nenten prasida kajantenang sajeroning sor singgihnyane sakewanten ring basa Bali Baru kruna 'sungsut' ngranjing ring Alus Singgih (Anom dkk, 2014). Lianan ring punika kruna basa Bali Kuna sane madue teges meluas taler ring basa Bali Baru madue kawigunan sane menyempit sakadi kruna 'aji' sane mateges 'bapa', manut pangweruhan panilik kruna punika yening ring basa Bali Kuna pinaka sebutan majeng ring rerema lanang, sakewanten ring basa Bali Baru kruna bapa punika lianan ngranjing ring sor singgih basa taler ngranjing sebutan rerama ring catur warna (sudra).

Manut sakadi teori sosiolinguistik paubahan teges menyempit inggih punika paubahan teges kruna sane dumun madue teges langkungan teken asiki nanging sane mangkin madue teges wantah asiki (Chaer, 2004: 141). Sajeroning tetilikan puniki kapolihang kosa kata basa Bali Kuna sane tegesnyane menyempit sajeroning basa Bali Baru minakadi, kruna sane wit tegesnyane tiga dados kalih wenten tiga, kruna sane wit tegesnyane tiga dados siki wenten kalih, miwah kruna sane wit tegesnyane kalih dados siki wenten asiki. Pikolih tetilikan sane sampun kamargiang paubahan teges menyempit kidikan ring paubahan teges meluas sajeroning prasasti mabasa Bali Kuna. Kruna basa Bali Kuna sane kantun kaanggen sajeronung basa Bali Baru madue teges menyempit wenten nenem kruna.

Kruna kulangkaling ring basa Bali Kuna madue teges 'nglindeng', 'buah jaka', miwah 'pesengan jadma' nanging ring basa Bali Baru madue teges 'buah jaka' kemanten. Sajeroning basa Bali Kuna kruna kulangkaling ketah kapanggihin mateges pesengan jadma sakadi ring punggelan prasasti Bantuja Al "nayakan makarun kulangkaling" sane mateges manggala persidangan sane mawasta kulangkaling. Sajeroning tetilikan Beratha nenten kapanggihin kruna kulangkaling.

Panureksan prasasti basa Bali Kuna warsa 915-942 Masehi miwah 951-982 Masehi ngamolihang data sane marupa teges kruna basa Bali Kuna miwah paubahan teges kosa kata basa Bali Kuna sajeroning basa Bali Baru. Paubahan teges kosa kata basa Bali Kuna sajeroning basa Bali Baru sane kalitikin wantah kalih minakadi paubahan teges sane meluas miwah paubahan teges sane menyempit. Sajeroning paubahan teges punika data sane ngranjing ring tetilikan wantah krunakruna basa Bali Kuna ring prasasti inucap sane kantun kanggen sajeroning basa Bali Baru. Kruna sane kapolihang sajeroning 
prasasti basa Bali Kuna sane kantun kaanggen ri kala mabebaosan ring basa Bali Baru sane madue paubahan teges meluas miwah menyempit prasida kapanggihin ring krama sajeroning mabebaosan sadina-dina, lian ring punika taler sampun ngranjing ring kamus BaliIndonesia Badan Pembina Bahasa Aksara dan Sastra Provinsi Bali.

\section{PAMUPUT}

Ringkesan ring tetilikan puniki pacang mahbahang indik (1) bantang pikobet. Bantang pikobet ring tetilikan puniki inggih punika teges kruna sajeroning prasasti warsa 915-942 Masehi miwah warsa 951983 Masehi ngeninin basa Bali Kuna sane kantun kanggen ring basa Bali Baru saha paubahan tegesnyane. (2) tetujon tetilikan. Tetujon sajeroning tetilikan puniki kaanutang ring makakalih bantang pikobet sane sampun kabahbahang. (3) kawigunan tetilikan. Kawigunan tetilikan puniki wenten kalih inggih punika kawigunan pamucuk (teoritis) miwah kawigunan panglimbak (praktis). (4) teori sane kaanggen. Teori sane kaanggen inggih punika sosiolinguistik terjemahan miwah Semantik. (5) kramaning tetilikan. Kramaning tetilikan sane kanggen inggih punika kramaning dokumentasi sane mapiranti antuk kartu data. (6) pikolih tetilikan. Pikolih tetilikan puniki marupa teges kruna basa Bali Kuna sane kantun kaanggen ring basa Bali Baru saha paubahan tegesnyane sane meluas miwah menyempit sajeroning prasasti warsa 915942 Masehi miwah warsa 951-983 Masehi.

Saking pikolih miwah tetepasan sane sampun kabahbahang ring ajeng, prasida katingkesang sakadi ring sor. Kruna-kruna basa Bali Kuna sajeroning prasasti warsa 915-942 Masehi miwah warsa 951-982 Masehi sane kantun kaanggen ring basa Bali Baru akehnyane 172 kruna. Makasami krunane punika sampun prasida kasinahang tegesnyane sajeroning basa Bali Kuna. Sesampune nyaihang tegesnyane ring basa Bali Baru kapolihang 92 kruna sane mateges tetep, 24 kruna sane berubah total, miwah sane kantun mapaiketan (meluas 47 kruna saha menyempit 6 kruna). Paubahan teges kosa kata basa Bali Kuna sajeroning basa Bali Baru wenten kalih, minakadi paubahan teges meluas miwah paubahan teges menyempit. Sajeroning panureksan sane sampun kamargiang, paubahan teges meluas ngamolihang data 47 kruna miwah 6 kruna paubahan teges menyempit. Kabaos paubahan teges meluas duaning wenten kruna sane wit tegesnyane asiki dados kalih wenten telung dasa dua, kruna sane wit tegesnyane asiki dados tiga wenten solas, kruna sane wit tegesnyane asiki dados patpat wenten kalih miwah kruna sane wit tegesnyane asiki dados lelima wenten asiki. Paubahan sajeroning sor singgih basa taler kabaos paubahan teges meluas duaning ring basa Bali Kuna dereng prasida kajantenang sor singgih basanyane, sakadi kruna 'aji' ring basa Bali Kuna mateges 'bapa' sajeroning basa Bali Baru kruna 'aji' mateges 'bapa', sakewanten ring basa Bali Baru kruna 'aji' punika sampun ngranjing ring alus singgih tur wantah prasida kaanggen ring warna tri wangsa.

Malarapan antuk pikolih sane sampun kabahbahang, wenten piteket-piteket sane jagi kabahbahang sakadi ring sor. Majeng ring pangwacen risampune ngwacen tetilikan puniki mangda prasida uning taler madue kaweruhan ngeninin basa Bali Baru sane ketah kanggen ri kala mabebaosan sarahina kari wenten makudang-kudang kruna basa Bali Kuna sane kanggen. Majeng ring pangajah basa Bali mangda akeh ngrereh referensi indik basa Bali utaminnyane basa Bali Kuna miwah basa Bali Baru mangda kaweruhan sayan nincap.

\section{KAPUSTAKAAN}

Anom, I Gusti Ketut, dkk. 2014. Kamus BaliIndonesia Beraksara Bali dan Latin. Denpasar: Badan Pembina Bahasa, Aksara, dan Sastra Bali

Bawa, I Wayan, dkk.1985. Studi Sejarah Bahasa Bali.Denpasar: Pemerintah Daerah Provinsi Daerah Tingkat 1 Bali.

Beratha, Ni Luh Sutjiati. 1992. "Evolution of Verbal Morphology in Balinese" (thesis). Canberra: Australian Nation University.

Chaer, Abdul miwah Leonie Agustina. 2010. Sosiolinguistik Perkenalan Awal. Jakarta: Rineka Cipta.

Goris, R. 1954. Prasasti Bali Jilid I. Bandung: N.V. Masa Baru. 
------. 1967. Ancient History of Bali. Denpasar: Universitas Udayana.

Granoka, Ida Wayan Oka dkk.1984. Kamus Bahasa Bali Indonesia.Denpasar:
Kuna-

Proyek
Pengembangan Bahasa dan Sastra Indonesia dan Daerah.

Hariyanto, Sugeng. 2003. Bahasa Teori dan Penuntun Praktis Menerjemahkan. Yogyakarta: Kanisius. 